Z. Wahrscheinlichkeitstheorie verw. Gebiete

$35,103-113(1976)$
Zeitschrift für

Wahrscheinlichkeitstheorie und verwandte Gebiete

(C) by Springer-Verlag 1976

\title{
Estimating a Mean from Delayed Observations
}

\author{
Norman Starr ${ }^{1 \star}$, Robert Wardrop ${ }^{2}$, and Michael Woodroofe ${ }^{1 \star}$ \\ 1 Statistics Department, University of Michigan, Ann Arbor, Mich. 48104, USA \\ 2 Department of Statistics, University of Wisconsin, Madison
}

\section{Section 1. Introduction}

We consider a class of estimation problems for which the collection of data is not entirely under our control, but depends also on an element of chance. In particular, we are interested in data which, no matter how we choose to manipulate our environment, is forthcoming only at random times.

For example, in studying the in situ effectiveness of experimental safety devices (such as passive air cushion restraints) relevant data may become available only as a result of accidents. Medical data (such as data on drug abuse or an asymptomatic disease) can sometimes only be obtained when patients voluntarily seek help or are somehow otherwise identified and examined, at random times. Other examples we have in mind are data collected in response to a mail survey or mailorder campaign, data concerning objects uncovered at random times at an archeological site, and data resulting from an undersea survey of containerized radioactive waste (see Dyer (1975), for example).

We will consider a relatively simple form of such problems - namely, the estimation of a normal mean. Let $y_{1}, \ldots, y_{n}$ be independent normally distributed random variables with unknown mean $\theta$ and known variance $\sigma^{2}$. We suppose that $y_{i}$ is observed at time $t_{i}$, where $0 \leqq t_{1} \leqq t_{2} \leqq \cdots \leqq t_{n}$, and $t_{1}, \ldots, t_{n}$ are independent of $y_{1}, \ldots, y_{n}$. We will, in fact, suppose that $t_{1}, \ldots, t_{n}$ are the order statistics of positive exchangeable random variables $x_{1}, \ldots, x_{n}$, which are independent of $y_{1}, \ldots, y_{n}$, so that

$$
k(t)=\sum_{i=1}^{n} I_{[0, t]}\left(x_{i}\right)
$$

denotes the number of observations which have been made by time $t \geqq 0$.

For the present suppose that we agree to take at least one observation and to estimate $\theta$ by the average of the observed $y$-values at the time when we stop. Suppose also that the loss due to estimation error is squared error loss and that

* Research supported in part by NSF GP 38487. 
it costs $c$ units to observe the process for unit time. If we observe the process for $t$ units of time, then the conditional expected loss, given $k(s), s \leqq t$, is

$$
\sigma^{2} k(t)^{-1}+c t \text {. }
$$

We are thus led to a stopping problem: find a stopping time $t$ which minimizes the expected value of (1.2).

In Sections 2 and 3 of this paper we will consider the more general problem of finding a stopping time to maximize

$$
E\{h[k(\tau)]-c \tau\}
$$

with respect to stopping times $\tau$. We obtain explicit solutions for a large class of possible $h$ and a large class of possible distributions for $x_{1}, \ldots, x_{n}$. In Section 4 we apply the results of Sections 2 and 3 to the estimation problem discussed above. In Section 5 we propose an adaptive strategy for the estimation problem. The adaptive strategy requires only minimal knowledge of the distribution of $x_{1}, \ldots, x_{n}$ and performs nearly as well as is possible when $n$ is large.

The problem of minimizing (1.3) with respect to stopping times $\tau$ is of independent interest. It has been considered by Taylor (1968), Starr (1970), Wardrop (1974), and Starr and Woodroofe (1974).

\section{Section 2. Optimal Stopping : Known $F$}

In this section we suppose that $x_{1}, \ldots, x_{n}$ are independent random variables with a common distribution function $F$. Further, we suppose that $F(0)=0$; that $F(t)>0$ for $t>0$; that $F$ is absolutely continuous with density $f$; and that $f$ is the right hand derivative of $F$ on $(0, \infty)$. We denote the class of such $F$ by $\mathscr{G}$.

Let $h$ be a given extended real values function on $E_{n}=\{0,1, \ldots, n\}$ for which $h(0)<\infty$ and $|h(k)|<\infty$ for $k \geqq 1$, and let

$$
z_{h}(t)=h[k(t)]-c t
$$

for $t \geqq 0$. We regard $z_{h}(t)$ as our payoff if we stop the process at time $t$. Let $y_{1}, \ldots, y_{n}$ be random variables (or vectors) which are independent of $x_{1}, \ldots, x_{n}$, and let

$$
\mathscr{F}_{t}=\sigma\left\{k(s), s \leqq t, y_{1}, \ldots, y_{k}(t)\right\}
$$

be the information which is available to us at time $t$. By a stopping time we will mean an extended random variable $\tau$ for which $0 \leqq \tau<\infty$ w.p. 1 and $\{\tau>t\} \in \mathscr{F}_{t}$ for all $t \geqq 0$. We seek a stopping time which will maximize

$$
V_{h}(\tau)=E\left\{z_{h}(\tau)\right\}
$$

with respect to $\tau$. We denote the supremum of $V_{h}(\tau)$ with respect to $\tau$ by $V_{h}(n, F)$.

Let $b=\sup \{t: F(t)<1\}$, and $\rho(z)=f(z) /[1-F(z)], 0 \leqq z<b$, denote the failure rate. It is easy to check that $k(t), 0 \leqq t<b$, is a non-stationary Markov chain with respect to $\mathscr{F}_{t}, 0 \leqq t<b$ and that its characteristic operator is given by

$$
A_{t} g(k)=(n-k) \rho(t)[g(k+1)-g(k)]
$$

for $k \in E_{n}$ and all real-valued functions $g$ on $E_{n}$. 
Theorem 2.1. Suppose that $h(k+1)-h(k)$ is non-increasing for $k \leqq n-1$ and that $F \in \mathscr{G}$ has a non-increasing failure rate. Then $V_{h}(\tau)$ is maximized by

$$
\tau_{h}=\inf \left\{t \geqq 0: A_{t} h[k(t)] \leqq c\right\} .
$$

Proof. The proof follows Ross (1971). Suppose first that $h(0)$ is finite. Then Dynkin's formula [Breiman (1968), p. 376] and a simple truncation argument show that

$$
V_{h}(\tau)-h(0)=E\left\{\int_{0}^{t}\left(A_{t} h[k(t)]-c\right) d t\right\}
$$

for all stopping times $\tau$. In particular, it follows from (2.3) that $V_{h}\left(\tau_{h}\right) \geqq h(0)>-\infty$, so that $E\left\{\tau_{h}\right\}<\infty$. Next observe that, by assumption, $A_{t} h[k(t)]$ is a non-increasing function of $t$. Thus, if $\tau$ is any other stopping time then

$$
V_{h}\left(\tau_{h}\right)-V_{h}(\tau)=\int_{\left\{\tau_{h}>\tau\right\}}\left\{\int_{\tau}^{\tau_{h}}\left(A_{t} h[k(t)]-c\right) d t\right\} d P+\int_{\left\{\tau>\tau_{h}\right\}}\left\{\int_{\tau_{h}}^{\tau}\left(A_{t} h[k(t)]-c\right) d t\right\} d P \geqq 0,
$$

so that $\tau_{h}$ is optimal.

If $h(0)=-\infty$ and $E\left\{t_{1}\right\}=\infty$, then $V_{h}(\tau)=-\infty$ for all $\tau$, so that $\tau_{h}$ is trivially optimal; and if $h(0)=-\infty$ and $E\left\{t_{1}\right\}<\infty$, then it suffices to maximize $V_{h}(\tau)$ with respect to $\tau$ for which $\tau \geqq t_{1}$ w.p. 1 . For such $\tau$, Dynkin's formula and a simple truncation argument yield

$$
V_{h}(\tau)-V_{h}\left(t_{1}\right)=E\left\{\int_{t_{1}}^{\tau}\left(A_{t} h[k(t)]-c\right) d t\right\}
$$

and the argument proceeds as above.

Example 2.1. If $F$ is an exponential distribution, say $F(t)=1-e^{-\alpha t}$ for $t \geqq 0, \tau_{h}=t_{r}$, where $r$ is the least integer $k$ for which $(n-k)[h(k+1)-h(k)] \leqq c \alpha^{-1}$, and the optimal payoff is

$$
V_{h}^{*}(n, \alpha)=h(r)-\sum_{k=1}^{r} c / \alpha(n-k+1) .
$$

Lemma 2.1. If $F \in \mathscr{G}$ and $G \in \mathscr{G}$ and $F(t) \leqq G(t)$ for all $t \geqq 0$, then $V(n, F) \leqq V(n, G)$ for all $n \geqq 1$.

Proof. There are independent random variables $u_{1}, \ldots, u_{n}$ which are uniformly distributed on $[0,1]$ and functions $f \geqq g$ for which $x_{i}=f\left(u_{i}\right)$ and $y_{i}=g\left(u_{i}\right)$ have distributions $F$ and $G$ respectively. See Lehmann (1959), page 73. The lemma follows easily.

Theorem 2.2. If $F$ has non-increasing (non-decreasing) failure rate and if $\alpha=\rho(0)$ is finite and positive, then $V(n, F) \leqq(\geqq) V_{h}^{*}(n, \alpha)$.

Proof. Let $G$ be the exponential distribution with parameter $\alpha$. If $F$ has nonincreasing (non-decreasing) failure rate, then $F(t) \leqq(\geqq) G(t)$ for all $t \geqq 0$. The theorem now follows easily from Lemma 2.1 .

For later reference, we mention the following extension of Lemma 2.1. 
Lemma 2.2. For $\delta>0$, let $W(n, F)$ be the supremum of $V_{h}(\tau)$ over all stopping times $\tau$ for which $\tau \leqq \delta$. If $F \in \mathscr{G}$ and $G \in \mathscr{G}$ and $F(t) \leqq G(t)$ for $0 \leqq t \leqq \delta$, then $W(n, F) \leqq W(n, G)$.

The proof involves showing that the functions $f$ and $g$ (in the proof of Lemma 2.1) may be so chosen that $f(u) \geqq g(u)$ when $f(u) \leqq \delta$, and this is easily accomplished.

\section{Section 3. Optimal Stopping: Bayesian Formulation}

In this section we suppose that $x_{1}, \ldots, x_{n}$ are conditionally independent and exponentially distributed with parameter $w$, given that $W=w$, where $W$ is a random variable which has the gamma distribution with parameters $\alpha$ and $\beta$. That is, $W$ has density

$$
f(w)=\Gamma(\alpha)^{-1} \beta^{\alpha} w^{\alpha-1} e^{-\beta w}
$$

for $w>0$. Such a model might be appropriate if $x_{1}, \ldots, x_{n}$ were exponentially distributed with an unknown parameter $w$, and if $w$ were given its conjugate prior distribution. It is easy to see that the conditional distribution of $W$ given $\mathscr{F}_{t}$ is again a gamma distribution, but with new parameters,

$$
\alpha_{t}=\alpha+k(t) \text { and } \beta_{t}=\beta+\sum_{j=1}^{k(t)} t_{j}+(n-k(t)) t .
$$

We denote the prior parameters of (3.1) by $\alpha_{0}$ and $\beta_{0}$, and $\alpha_{0}+n$ by $m$. It is then easy to check that $\left(\alpha_{t}, \beta_{t}\right), t \geqq 0$, is a stationary Markov process with characteristic operator

$$
A f(\alpha, \beta)=(m-\alpha) f^{\prime}(\alpha, \beta)+\beta^{-1} \alpha(m-\alpha)[f(\alpha+1, \beta)-f(\alpha, \beta)]
$$

where ' denotes differentiation with respect to $\beta$. The domain of $A$ includes all $f$ which are continuously differentiable in $\beta$ for each $\alpha$.

As in the previous section, we let $h$ be a non-decreasing function on $\left\{\alpha_{0}, \ldots, m\right\}$. We require that $|h(\alpha)|<\infty$ for $\alpha>\alpha_{0}$ but allow the possibility that $h\left(\alpha_{0}\right)=-\infty$. We also let

$$
V_{h}(\tau)=E\left\{h\left(\alpha_{\tau}\right)-\tau\right\}
$$

for stopping times $\tau$.

Theorem 3.1. Suppose that $\alpha(m-\alpha)[h(\alpha+1)-h(\alpha)]$ is non-increasing for $\alpha=$ $\alpha_{0}, \ldots, m-1$. Then $V_{h}(\tau)$ is maximized by

$$
\tau_{h}=\inf \left\{t \geqq 0: A h\left(\alpha_{t}, \beta_{t}\right) \leqq c\right\} .
$$

The proof of Theorem 3.1 is similar to that of Theorem 2.1 and will be omitted. We observe that $\tau_{h}$ may be written in the form

$$
\tau_{h}=\inf \left\{t \geqq 0:(n-k(t))\left[h\left(\alpha_{t}+1\right)-h\left(\alpha_{t}\right)\right] w_{t} \leqq c\right\} .
$$

where $w_{t}=E\left\{W \mid \widetilde{F_{t}}\right\}$ is the Bayes estimate of $W$ at time $t$. (cf. (2.2)) 
Examples. i) If $h(\alpha)=-\alpha^{-1}$, then the condition of Theorem 3.1 is satisfied, since $\alpha(m-\alpha)[h(\alpha+1)-h(\alpha)]=(m-\alpha) /(\alpha+1)$. This example will arise in the next section.

ii) If $h(\alpha)=\alpha$, then the condition of Theorem 3.1 is satisfied if, and only if, $\alpha_{0}>n / 2$.

Let $V_{h}\left(\alpha_{0}, \beta_{0}\right)=\sup V_{h}(\tau)$ denote the optimal payoff. If $c=1$ and the hypotheses of Theorem 3.1 are satisfied then we may compute $V_{h}$ explicitly. Let

$$
\gamma_{\alpha}=\alpha(m-\alpha)[h(\alpha+1)-h(\alpha)]
$$

so that the continuation region for the optimal rule $\tau_{h}$ is

$$
\mathscr{C}=\left\{(\alpha, \beta): \beta<\gamma_{\alpha}\right\} \text {. }
$$

If $h\left(\alpha_{0}\right)>-\infty$, then $V_{h}$ satisfies the equation

$$
\begin{array}{ll}
A V_{h}(\alpha, \beta)=0 & (\alpha, \beta) \in \mathscr{C}, \\
V_{h}(\alpha, \beta)=h(\alpha) & \beta \geqq \gamma_{\alpha} .
\end{array}
$$

Theorem 3.2. Suppose that hypotheses of Theorem 3.1 are satisfied and that $h\left(\alpha_{0}\right)>$ $-\infty$. Then

$$
V_{h}(\alpha, \beta)=h(\alpha+k+1)+\beta^{\alpha} \sum_{j=0}^{k} c_{k, j}(\alpha)\left[\gamma_{\alpha+k}-\beta\right]^{j}
$$

for $\gamma_{\alpha+k+1} \leqq \beta<\gamma_{\alpha+k}$ and $k=0, \ldots, m-\alpha-1$ and $\alpha \leqq m-1$. Here the coefficients $c_{k, j}$ are defined inductively by

and

$$
c_{k, 0}(\alpha)=\gamma_{\alpha+k}^{-\alpha}[h(\alpha+k)-h(\alpha+k+1)]+\sum_{j=0}^{k-1} c_{k-1, j}(\alpha)\left[\gamma_{\alpha+k-1}-\gamma_{\alpha+k}\right]^{j}
$$

$$
c_{k, j}(\alpha)=\alpha j^{-1} c_{k-1, j-1}(\alpha+1)
$$

for $j=1, \ldots, k$.

Proof. Let $g_{\alpha}(y)=y^{-\alpha} V_{h}(\alpha, y)$ for $y>0$ and $\alpha=\alpha_{0}, \ldots, m$. Then

$$
g_{\alpha}^{\prime}(y)=-\alpha g_{\alpha+1}(y)
$$

for $0<y<\gamma_{\alpha}$ and $\alpha \leqq m-1$ and $g_{m}(y)=h(m) y^{-m}$ for $y>0$. Thus, for $\gamma_{\alpha+k+1} \leqq y<\gamma_{\alpha+k}$

$$
g_{\alpha}(y)=g_{\alpha}\left(\gamma_{\alpha+k}\right)+\int_{y}^{\gamma_{\alpha+k}} \alpha g_{\alpha+1}(u) d u .
$$

The theorem now follows by a straightforward, if tedious, induction.

\section{Section 4. Estimation with Delayed Observations}

In this section we consider the problem of estimating the mean of a normal distribution when the observations become available at random times. Thus, let $y_{1}, \ldots, \mathrm{y}_{n}$ be independent normally distributed random variables with unknown 
mean $\theta$ and known variance $\sigma^{2}$. We suppose that $y_{i}$ is observed at time $t_{i}, i=1, \ldots, n$, where $t_{1}, \ldots, t_{n}$ are the order statistics of positive exchangeable random variables $x_{1}, \ldots, x_{n}$ which are independent of $y_{1}, \ldots, y_{n}$. We suppose further that we may observe the process as long as we please: that when we stop, we must report an estimate $\hat{\theta}$ of $\theta$; and that if we stop at time $t$ and report the estimate $\hat{\theta}$, we incur the loss

$$
L_{t}=(\hat{\theta}-\theta)^{2}+c t
$$

where $c>0$.

We will first adopt a Bayesin approach by placing a prior distribution over $\theta$. More formally, we invent a random variable $\Theta$ with distribution $\pi$ and suppose that conditionally given $\Theta=\theta, y_{1}, \ldots, y_{n}$ are independent normal random variables with mean $\theta$ and variance $\sigma^{2}$ and that $\left(\Theta, y_{1}, \ldots, y_{n}\right)$ are independent of $x_{1}, \ldots, x_{n}$. We denote unconditional probability by $P^{\pi}$ and conditional probability given $\Theta=\theta$ by $P_{\theta}$, and we suppose that $E^{\pi}\left\{\Theta^{2}\right\}<\infty$.

Let $\mathscr{F}_{t}=\sigma\left\{k(s), s \leqq t, y_{1}, \ldots, y_{k(t)}\right\}$ be the information available to us at time $t$, as in (2.1). By a strategy, we will understand a pair $\delta=(\hat{\theta}, \tau)$, where $\tau$ is a stopping time and $\hat{\theta}$ is an $\mathscr{\mathscr { F }}_{t}$-measurable random variable. The risk function and Bayes' risk of the strategy $\delta$ are then defined by

$$
r(\delta, \theta)=E_{\theta}\left\{L_{\tau}\right\} \text { and } \bar{r}(\delta, \pi)=E^{\pi}\left\{L_{\tau}\right\}
$$

respectively.

It is clear that for any stopping time $\tau, \bar{r}(\delta, \pi)$ is minimized by letting $\hat{\theta}=$ $E\left\{\Theta \mid \mathscr{F}_{\tau}\right\}$ be the conditional expectation of $\Theta$ given $\mathscr{F}_{\tau}$. Thus, the problem of finding a Bayes' rule may be reduced to an optimal stopping problem. In the special case that $\pi$ is a normal distribution, the stopping problem is of the form considered in Sections 2 and 3.

Lemma 4.1. Suppose that $\pi$ is the normal distribution with mean $m_{0}$ and variance $\sigma_{0}^{2}$. If $\tau$ is any stopping time then the conditional distribution of $\Theta$ given $\mathscr{F}_{\tau}$ is normal with mean

$$
m_{\tau}=\left[\sigma_{0}^{-2} m_{0}+\sigma^{-2}\left(y_{1}+\cdots+y_{k(\tau)}\right)\right] /\left(\sigma_{0}^{-2}+k(\tau) \sigma^{-2}\right)
$$

and variance $1 /\left(\sigma_{0}^{-2}+k(\tau) \sigma^{-2}\right)$.

Proof. When $\tau$ is a constant, the lemma follows directly from Bayes' theorem. The transition from constants to stopping times then follows from the Strong Markov property.

Thus, if $\pi$ is the normal distribution with mean $m_{0}$ and variance $\sigma_{0}^{2}$, then the problem of finding a Bayes' rule reduces to the problem of minimizing

$$
U^{\pi}(\varepsilon, \tau)=E^{\pi}\left\{\sigma^{2}(\varepsilon+k(\tau))^{-1}+c \tau\right\}
$$

with respect to $\tau$, where $\varepsilon=\sigma^{2} / \sigma_{0}^{2}$. If $x_{1}, \ldots, x_{n}$ are independent with a common distribution function $F \in \mathscr{G}$, and if $F$ has a non-increasing failure rate, this is a special case of the problem which was considered in Section 2; and it follows from Theorem 2.1 that for any prior $\pi$ (not just normal priors) $U^{\pi}(\varepsilon, \tau)$ is minimized by

$$
\tau_{\varepsilon}=\inf \left\{t \geqq 0:[n-k(t)] \rho(t) \leqq c \sigma^{-2}[\varepsilon+k(t)][\varepsilon+k(t)+1]\right\} .
$$


It also follows from Theorem 2.1 that $U^{\pi}\left(\varepsilon, \tau_{\varepsilon}\right)$ is independent of $\pi$. As a consequence, we have

Theorem 4.1. Suppose that $F \in \mathscr{G}$ has non-increasing failure rate $\rho$. If $\pi_{\varepsilon}$ is the normal distribution with mean $m_{0}$ and variance $\sigma_{0}^{2}=\sigma^{2} / \varepsilon$, where $0<\varepsilon<\infty$, then the Bayes' strategy is $\delta_{\varepsilon}=\left(m_{\tau_{\varepsilon}}, \tau_{\varepsilon}\right)$.

It seems natural to ask whether the strategy $\delta_{0}$ is minimax. The answer is provided by

Theorem 4.2. If $E\left\{t_{1}\right\}<\infty$, then $\delta_{0}$ is minimax.

Proof. When $\pi$ is degenerate at 0 , we will write $U$ for $U^{\pi}$. Then, as remarked above, $\bar{r}\left(\delta_{\varepsilon}, \pi_{\varepsilon}\right)=U\left(\varepsilon, \tau_{\varepsilon}\right)$. Also, it is easy to see that $r\left(\delta_{0}, \theta\right)=U\left(0, \tau_{0}\right)$ is a finite constant. Thus, it will suffice to show that

$$
U\left(\varepsilon, \tau_{\varepsilon}\right) \rightarrow U\left(0, \tau_{0}\right)
$$

as $\varepsilon \rightarrow 0$. See, for example, Ferguson (1967), page 90. To establish (4.1), let $\tau_{\varepsilon}^{*}=$ $\max \left\{t_{1}, \tau_{\varepsilon}\right\}$. Then, since $\tau_{0}$ is optimal, we have $U\left(0, \tau_{0}\right) \leqq U\left(0, \tau_{\varepsilon}^{*}\right)$, so that

$$
0 \leqq U\left(0, \tau_{0}\right)-\bar{r}\left(\delta_{\varepsilon}, \pi_{\varepsilon}\right) \leqq U\left(0, \tau_{\varepsilon}^{*}\right)-U\left(\varepsilon, \tau_{\varepsilon}\right) \leqq \varepsilon \sigma^{2}+\int_{\left\{\tau_{\varepsilon}^{*}>\tau_{\varepsilon}\right\}} c\left(\tau_{\varepsilon}^{*}-\tau_{\varepsilon}\right) d P_{0}
$$

Now the event $\left\{\tau_{\varepsilon}^{*}>\tau_{\varepsilon}\right\}$ can occur only if $\tau_{\varepsilon}<t_{1}$ in which case $\tau_{\varepsilon}^{*}-\tau_{\varepsilon} \leqq t_{1}-\tau_{\varepsilon}$. Thus, since $E\left\{t_{1}\right\}<\infty$, it will suffice to show that $\lim P_{0}\left\{\tau_{\varepsilon}>t_{1}\right\}=0$ as $\varepsilon \rightarrow 0$. Since

$$
\varepsilon^{-1} P_{0}\left\{t_{1}>\tau_{\varepsilon}\right\} \leqq U\left(\varepsilon, \tau_{\varepsilon}\right) \leqq U\left(0, \tau_{0}\right)<\infty
$$

for $\varepsilon>0$, the theorem follows.

As a corollary to Theorem 4.2 it is easily seen that the strategy $\delta_{0}$ is best among all strategies which are invariant with respect to translations of $y_{1}, \ldots, y_{n}$. Indeed, $\delta_{0}$ is invariant, and any invariant rule has a constant risk function.

One may formulate a similar result if $x_{1}, \ldots, x_{n}$ are conditionally independent exponential random variables with unknown parameter $w$ and $w$ has the (conjugate prior) gamma distribution with parameters $\alpha>0$ and $\beta>0$, as in Section 3 . Let

$$
\tau_{\varepsilon}=\inf \left\{t \geqq 0:\left(m-\alpha_{t}\right) \alpha_{t} \beta_{t}^{-1} \leqq c \sigma^{-2}[\varepsilon+k(t)][\varepsilon+k(t)+1]\right.
$$

for $\varepsilon>0$, where $\alpha_{t}$ and $\beta_{t}$ are as in (3.2).

Theorem 4.3. Suppose that the distribution of $x_{1}, \ldots, x_{n}$ is as described in Section 3. Then for sufficiently small $\varepsilon>0, \delta_{\varepsilon}=\left(m_{\tau_{\varepsilon}}, \tau_{\varepsilon}\right)$ is Bayes' with respect to $\pi_{\varepsilon}$.

Proof. The proof of Theorem 4.3 is similar to that of Theorem 4.1 and will be omitted.

\section{Section 5. An Adaptive Rule}

The strategies developed in Theorem 4.1 and 4.2 require that $F$, the common distribution function of $x_{1}, \ldots, x_{n}$, be known exactly (and have a non-increasing failure rate). The strategy of Theorem 4.3 allows $F$ to be unknown, but requires 
it to be an exponential distribution. In this section we will develop an adaptive strategy which requires knowledge of neither $n$ nor $F$ and performs nearly as well as is possible when $n$ is large for a large class of $F$.

As in Section 4 , let $y_{1}, \ldots, y_{n}$ be independent random variables which are independent of $x_{1}, \ldots, x_{n}$ and have a common normal distribution with unknown mean $\theta \in R$ and known variance $\sigma^{2}>0$. In this section we will only consider strategies $\sigma=(\hat{\theta}, \tau)$ for which $\tau \geqq t_{1}$ and $\hat{\theta}=\left(y_{1}+\cdots+y_{k(\tau)}\right) / k(\tau)$. The minimum risk for such strategies is then

$$
V(n, F)=\inf E\left\{\sigma^{2} k(\tau)^{-1}+c \tau\right\},
$$

where the infimum extends over all stopping times (with respect to $\mathscr{F}_{t}=\sigma\{k(s)$, $\left.\left.s \leqq t, y_{1}, \ldots, y_{k(t)}\right\}\right)$.

Theorem 5.1. Suppose that $F \in \mathscr{G}$ has a failure rate $\rho$ which is positive and continuous on $[0, \eta)$ for some $\eta>0$. Let $\alpha=\rho(0)$ and $a=a_{F}=c / \alpha$. Then

$\liminf \sqrt{n} \cdot V(n, F) \geqq 2 \sigma \sqrt{a}$

as $n \rightarrow \infty$.

Proof. Let $0<\varepsilon<\alpha$ be given and let $\delta>0$ be so small that $|\rho(t)-\alpha|<\varepsilon$ for $0 \leqq t \leqq \delta$. Also let $h(k)=\sigma^{2} /(k+1)$ for $k=0, \ldots, n$. If $\tau$ is any stopping time and if $\tau_{\delta}=$ $\min \{\tau, \delta\}$, then

$$
\begin{aligned}
E\left\{\sigma^{2} k(\tau)^{-1}+c \tau\right\} & \geqq E\{h[k(\tau)]+c \tau\} \\
& \geqq E\left\{h\left[k\left(\tau_{\delta}\right)\right]+c \tau_{\delta}\right\}-E\{h[k(\delta)]\} .
\end{aligned}
$$

It is easy to see that $E\{h[k(\delta)]\}=O\left(n^{-1}\right)$ as $n \rightarrow \infty$. Thus, letting $W(n, F)$ be the infimum of $E\{h[k(\tau)]+c \tau\}$ over all stopping times $\tau \leqq \delta$, we find that

$$
V(n, F) \geqq W(n, F)-O\left(n^{-1}\right) \text {. }
$$

Next let $G(t)=1-\exp \{-(\alpha+\varepsilon) t\}$ for $t \geqq 0$, so that $F(t) \leqq G(t)$ for $0 \leqq t \leqq \delta$. Then $W(n, F) \geqq W(n, G)$, by Lemma 2.2 , so that

$$
V(n, F) \geqq V(n, G)-O\left(n^{-1}\right) \text {. }
$$

Finally, it follows from Example 2.1 that $\lim \sqrt{n} \cdot V(n, G)=2 \sqrt{a^{\prime}}$, where $a^{\prime}=$ $c /(\alpha+\varepsilon)$, and the theorem follows by letting $\varepsilon \rightarrow 0$.

Let us now consider the adhoc strategy which terminates sampling at time

$$
\tau_{0}=\inf \left\{t \geqq 0: t k(t) \geqq c^{-1} \sigma^{2}\right\}
$$

and estimates $\theta$ with $\hat{\theta}=\left[y_{1}+\cdots+y_{k\left(\tau_{0}\right)}\right] / k\left(\tau_{0}\right)$. Observe that this strategy requires knowledge of neither $n$ nor $F$ for its implementation. Let

$$
V_{0}(n, F)=E\left\{\sigma^{2} k\left(\tau_{0}\right)^{-1}+c \tau_{0}\right\}
$$

be the resulting risk. We will now show that $V_{0}(n, F) \sim V(n, F)$ as $n \rightarrow \infty$, provided that the following additional condition is satisfied: for some $m \geqq 1$,

$$
\int_{0}^{\infty}[1-F(t)]^{m} d t<\infty .
$$

Of course, if (5.2) fails for all $m$, then $V(n, F)=\infty$ for all $n$. 
Theorem 5.2. Suppose that the hypotheses of Theorem 5.1 are satisfied and that (5.2) holds. Then

$$
\lim \sqrt{n} V_{0}(n, F)=2 \sigma \sqrt{a}
$$

as $n \rightarrow \infty$.

Proof. By Theorem 5.1, it will suffice to show that $\lim \sup \sqrt{n} \cdot V_{0}(n, F) \leqq 2 \sigma \sqrt{a}$; and it will suffice to show this in the special case that $\sigma^{2}=1$.

Observe first that $k\left(\tau_{0}\right)^{-1} \leqq c \tau_{0}$, so that $V_{0}(n, F) \leqq 2 c E\left\{\tau_{0}\right\}$. Let $\tau_{n}=\sqrt{n} \tau_{0}$ and $K_{n}(s)=k(s / \sqrt{n})$ for $s \geqq 0$. Then

$$
P\left\{\tau_{n}>s\right\}=P\left\{K_{n}(s)<\frac{\sqrt{n}}{c s}\right\}
$$

for $s>0$. Since $K_{n}(s)$ has the binomial distribution with parameters $n$ and $p=$ $F(s / \sqrt{n})$, it is easily seen that $K_{n}(s) / \sqrt{n} \rightarrow \alpha s$ in probability as $n \rightarrow \infty$ for each $s>0$. From this it follows that the right side of (5.3) tends to 1 if $\alpha s<1 / c s$ and tends to 0 if $\alpha s>1 / c s$. That is,

$$
\tau_{n} \rightarrow 1 / \sqrt{\alpha c}=s_{0},
$$

say, in probability. Thus, it will suffice to show that $\tau_{n}, n \geqq 1$, are uniformly integrable.

To show uniform integrability, write (for $n$ sufficiently large)

$$
\begin{aligned}
\int_{\left\{\tau_{n}>2 s_{0}\right\}} \tau_{n} d P & =\int_{\left\{\tau_{n}>2 s_{0}\right\}}\left(\tau_{n}-2 s_{0}\right) d P+o(1) \\
& =\int_{2 s_{0}}^{c^{-1} \sqrt{n}} G_{n}(s) d s+\int_{c^{-1} \sqrt{n}}^{\infty} G_{n}(s) d s+o(1),
\end{aligned}
$$

where $G_{n}(s)=P\left\{\tau_{n}>s\right\}$. For $s \geqq c^{-1} \sqrt{n}, G_{n}(s)=P\left\{K_{n}(s)=0\right\}=[1-F(s / \sqrt{n})]^{n}$, so the second integral in (5.4) does not exceed

$$
\sqrt{n}\left[1-F\left(\frac{1}{c}\right)\right]^{n-m} \int_{0}^{\infty}[1-F(s)]^{m} d s=o(1) .
$$

To estimate the first, we use Bernstein's inequality. For fixed $x \geqq 2 s_{0}$ and $t>0$,

$$
\begin{aligned}
P\left\{K_{n}(s)<\frac{\sqrt{n}}{c s}\right\} & \leqq \exp \left\{\frac{t \sqrt{n}}{c s}\right\}\left[1-F\left(\frac{s}{\sqrt{n}}\right)\left(1-e^{-t}\right)\right]^{n} \\
& \leqq \exp \left\{\frac{t \sqrt{n}}{c s}-n F\left(\frac{s}{\sqrt{n}}\right)\left(1-e^{-t}\right)\right\} .
\end{aligned}
$$

When $s=2 s_{0}$ and $t$ sufficiently small, it is easy to see that the last line of (5.5) is $o\left(\sqrt{n^{-1}}\right)$. Thus, the first integral on the right side of (5.4) also tends to 0 as $n \rightarrow \infty$. This completes the proof.

Corollary 5.1. If the hypotheses of Theorem 5.2 are satisfied, then

$$
\lim \sqrt{n} \cdot V(n, F)=2 \sigma \sqrt{a}
$$

as $n \rightarrow \infty$. 


\section{Section 6. Extensions}

1. In Section 2, it can be shown that if $F \in \mathscr{G}$ and $h(0)>-\infty$, then an optimal rule exists and is given by

$$
\tau=\inf \left\{t \geqq 0: h[k(t)] \leqq W\left[n, k(t), F_{t}\right]\right\}
$$

where $W(n, k, F)=V_{h(k+\cdot)}(n-k, F)$ and $F_{t}(s)=[F(s+t)-F(t)] /[1-F(t)]$.

2. Theorem 2.1 may be generalized by replacing $c t$ by $c(t)$, provided that $c$ is convex. The optimal rule is then

$$
\tau_{h}=\inf \left\{t \geqq 0: A_{t} h[k(t)] \leqq c^{\prime}(t)\right\} .
$$

A similar remark applies to Theorem 3.1.

3. In the context of Theorem 4.2, it can be shown that if $E\left(t_{1}^{2}\right)<\infty$, then $\delta_{0}$ is admissible. Indeed, by (4.2), $\mathrm{P}_{0}\left\{t_{1}>\tau_{\varepsilon}\right\}=O(\varepsilon)$, so

$$
\int_{\left\{\varepsilon_{\varepsilon}^{*}>\tau_{\varepsilon}\right\}} c\left(\tau_{\varepsilon}^{*}-\tau_{\varepsilon}\right) d P \leqq \sqrt{P_{0}}\left\{t_{1}>\tau_{\varepsilon}\right\} \cdot V \int_{\left\{t_{1}>\tau_{\varepsilon}\right\}} c^{2} t_{1}^{2} d P_{0}=o(\sqrt{\varepsilon})
$$

as $\varepsilon \rightarrow 0$, if $E\left\{t_{1}^{2}\right\}<\infty$. Admissibility now follows from, for example, Farrell (1968).

4. It is possible to generalize Theorem 4.1 to the case that $L(\theta, \hat{\theta})=|\theta-\hat{\theta}|^{\alpha}+c(t)$, where $\alpha>0$ and $c$ is convex.

5. Suppose that the assumptions of Section 4 remain in force, except we assume that given $\Theta=\theta, y_{1}, \ldots, y_{n}$ are i.i.d. with Bernoulli distribution defined by $P\left(y_{i}=1\right)=\theta=1-P\left(y_{i}=0\right)$ for $i=1, \ldots, n$; that $\Theta$ has a prior distribution $\pi$ which is uniform on $(0,1)$; and that for any stopping time $\tau$, the risk is given by

$$
r(\delta, \theta)=E_{\theta}\left\{\frac{(\hat{\theta}-\theta)^{2}}{\theta(1-\theta)}+c \tau\right\} .
$$

If $F \in \mathscr{G}$ has non-increasing failure rate $\rho$, then $\delta_{0}=\left(m_{\tau_{0}}, \tau_{0}\right)$ is Bayes with respect to $\pi$ and minimax, where

and

$$
m_{\tau_{0}}=\frac{y_{1}+\cdots y_{k\left(\tau_{0}\right)}}{k\left(\tau_{0}\right)}
$$

$$
\tau_{0}=\inf \{t \geqq 0:(n-k(t)) \rho(t) \leqq c k(t)(k(t)+1)\} .
$$

\section{References}

Breiman, L.: Probability. Reading, Massachusetts: Addison Wesley 1968

Dyer, R.S.: Ocean disposal of radioactive wastes, EPA Journal, U.S. Environmental Protection Agency 1 (1975)

Farrell, R.H.: On a necessary and sufficient condition for admissibility of estimators when strictly convex loss is used. Ann. Math. Statist. 39, 23-28 (1968)

Ferguson, T.: Mathematical Statistics. New York: Academic Press 1967

Lehmann: Testing Statistical Hypotheses. New York: Wiley 1959 
Ross, S.: Infititesmal look-ahead stopping rules. Ann. Math. Statist. 42, 297-303 (1971)

Starr, N.: Optimal and adaptive stopping based on capture times. J. App. Probab. 11, 294-301 (1974)

Starr, N., Woodroofe, M.: Gone fishin': optimal stopping based on catch times. Technical Report No. 33, Department of Statistics, The University of Michigan, Ann Arbor (1974)

Taylor, H.: Optimal stopping in a Markov process. Ann. Math. Statist. 39, 1333-1344 (1968)

Wardrop, R.: Optimal stopping based on capture times; the increasing failure rate case. Ph. D. dissertation, Department of Statistics, The University of Michigan, Ann Arbor (1974)

Received November 22, 1975 